\title{
RECURRENT POINTS AND DISCRETE POINTS FOR ELEMENTARY AMENABLE GROUPS
}

\author{
MOSTAFA NASSAR \\ Jodrey School of Computer Science \\ Acadia University \\ Wolfville, Nova Scotia \\ B0P 1X0 Canada
}

(Received January 18, 1989 and in revised form March 31, 1992)

ABSTRACT. Let $\beta G$ be the Stone-Cech compactification of a group $G, A^{G}$ the set of all almost periodic points in $\beta G, K^{G}=c \ell\left[\cup\left\{\operatorname{supp} \mu_{\varphi}: \varphi \in \operatorname{LIM}(G)\right\}\right]$ and $R^{G}$ the set of all recurrent points in $\beta G$. In this paper we will study the relationships between $K^{G}$ and $R^{G}$, and between $A^{G}$ and $R^{G}$. We will show that for any infinite elementary amenable group $G, A^{G} \varsubsetneqq R^{G}$ and $R^{G}-K^{G} \neq \phi$.

Key words and Phrases: Topological group, recurrent point, elementary amenable groups.

1980 AMS subject classification code. 43A60.

\section{INTRODUCTION}

Let $G$ be a discrete group, $M(G)$ the Banach space of all bounded real valued functions on $G$ with the supremum norm, and $M(G)^{*}$ the conjugate Banach space on $M(G)$. A group $G$ is called left amenable if there exists a mean on $M(G)$ which is left invariant [4]. Denote by $\operatorname{LIM}(G)$ the set of left invariant means on $M(G)$. By using Riesz representation theorem, then there exists a bounded regular Borel measure $\mu_{\varphi}$ on $\beta S$ such that

$$
\varphi(f)=\int_{\beta S} \bar{f} d \mu_{\varphi}, f \epsilon M(G) .
$$

For a subset $H$ of a left amenable group $G$ define the upper density as follows:

$$
\bar{d}(H)=\sup \left\{\varphi\left(\chi_{H}\right): \varphi \epsilon \operatorname{LIM}(G)\right\}=\sup \left\{\mu_{\varphi}(\bar{H}): \varphi \epsilon \operatorname{LIM}(G)\right\}
$$

where $\chi_{H}$ is the characteristic function of $H$.

$$
\text { Put } K^{G}=c \ell\left[\cup\left\{\operatorname{supp} \mu_{\varphi}: \varphi \epsilon \operatorname{LIM}(G)\right\}\right]
$$




\section{DEFINITIONS:}

(1) For each $A \subset G$, let $\hat{A}=c \ell_{\beta G} A-G$. The set $\hat{A}$ is closed and open in $\hat{G}=\beta G-G$. Then a point $w \epsilon \hat{G}$ is said to be almost periodic if for every neighbourhood $U$ of $w$ there exist $A, K \subset G$ with $K$ finite, $A w \subset \cup$, and $G=K A$. Denote by $A^{G}$ the set of all almost periodic points in $\beta G$.

(2) $w \epsilon \beta G$ is recurrent if whenever $\cup$ is a neighbourhood of $w$ then the set $\{x \epsilon G: x w \epsilon \epsilon$ is infinite. Let $R^{G}$ be the set of all recurrent points in $\beta G$.

(3) $w \epsilon \beta G$ is discrete if its orbit $0(w)$ is discrete with respect to the subspace topology of $\beta G$. Let $D^{G}$ be the set of all such points in $\beta G$.

(4) $w \epsilon \beta G$ is strongly discrete if there exists a neighbourhood $U$ of $w$ such that $x \cup \cap y \cup=\phi$ where $x, y \epsilon G, x \neq y$. The set of all strongly discrete points will be denoted by $D S^{G}$. Let $W R^{G}=\beta G-S D^{G}$, the set of all weak recurrent points of $\beta G$.

If $G$ is a finite group, then $A^{G}=D^{G}=K^{-G}=G=\beta G$ and $R^{G}=W R^{G}=\phi$. Therefore, we are only interested in infinite groups. For the remainder of this paper, $G$ stands for an infinite group.

LEMMA 1.1. (1) $A^{G} \subset R^{G} \subset W R^{G}, S D^{G} \subset D^{G}$ and $D^{G}=\beta G-R^{G}$.

(2) If $G$ is amenable, then $A^{G} \subset K^{G} \subset W R^{G}$. [4]

As mentioned in Day [3], the family of amenable groups is closed under the following four standard processes of constructing new groups from given ones: (a) subgroup, (b) factor group, (c) group extension, (d) expanding union (or direct limit). Denote by EG the family of elementary amenable groups. It is the smallest family of groups containing all finite groups and all abelian groups, and is closed under (a) - (d).

As pointed out by Chou [2], the groups in EG can be constructed from abelian groups and finite groups by applying processes (c) and (d) only. Moreover, every periodic group in $E G$ is locally finite, and since it is known that every infinite locally finite group contains an infinite abelian subgroup (Robinson [5; p. 95]), we have the following:

LEMMA 1.2 (Chou). If $G$ is an infinite group in $E G$, then $G$ contains an infinite abelian subgroup. 


\section{$2 \cdot R_{0}$ - SETS AND RECURRENT POINTS:}

In this section we will show that $A^{G} \varsubsetneqq R^{G}$ and $R^{G}-K^{G} \neq \phi$, for any infinite elementary amenable group $G$.

DEFINITION. A subset $A$ of an amenable group $G$ is called $R_{0}$-set if:

$$
\begin{aligned}
& \left(R_{0} \cdot 1\right) \quad \bar{d}(A)=0, \text { and } \\
& \left(R_{0} \cdot 2\right) \quad \bar{A} \cap R^{G} \neq \phi .
\end{aligned}
$$

PROPOSITION 2.1: If an amenable group $G$ contains an $R_{0}$-set, then $A^{G} \varsubsetneqq R^{G}$ and $R^{G}-K^{G} \neq \phi$.

PROOF: If $A$ is an $R_{0}$-set in $G$, then by $\left(R_{0} \cdot 2\right)$ there exists $w \epsilon \bar{A} \cap R^{G}$ since $\bar{d}(A)=0, w \notin K^{G}$. The proof is completed.

$R_{0}$-sets were first studied by Chou for the case $G=Z$ where he showed that $Z$ contains such sets (see Chou [1], P. 60, example 2). In this section we will show that every finite elementary amenable group contains $R_{0}$-sets.

THEOREM 2.2: Let $\left\{A_{k}\right\}$ be a sequence of subsets of a group $G$, and $\left\{g_{k}\right\}$ be a sequence of different elements of $G$ such that

1. $A_{1} \supset A_{2} \supset A_{3} \supset \ldots$, and

2. $g_{n} A_{n+1} \subset A_{n}$ for all $n$. Then $\bigcap_{n=1}^{\infty} \bar{A}_{n}$ contains a recurrent point.

PROOF. Let $\pi$ be the family of sequences of closed subsets of $\hat{G}$. Let $\pi$ be defined as follows: a sequence of closed subsets $\left\{F_{n}\right\}$ of $\hat{G}$ belongs to $\pi$ if for each $n \epsilon N$,

(i) $F_{n} \subset \hat{A}_{n}$,

(ii) $F_{n+1} \subset F_{n}$,

(iii) $g_{n} F_{n+1} \subset F_{n}$, and

(iv) $F_{n} \neq \phi$.

Note that $\pi$ is non-empty since $\left\{\hat{A}_{n}\right\} \epsilon \pi . \pi$ can be ordered in the following natural way: $\left\{F_{n}\right\} \leq\left\{F_{n}^{\prime}\right\}$ if and only if $F_{n} \subset F_{n}^{\prime}$ for each $n$. It is easy to check that each chain in $\pi$ has a lower bound. Then using Zorn's lemma, $\pi$ has a minimal element $\left\{K_{n}\right\}$. Let $w \epsilon \bigcap_{n=1}^{\infty} K_{n}$. We claim that $w$ is a recurrent point, by showing that if $\hat{A}$ is an open neighbourhood of $w$, then there exists infinitely many $g \epsilon G$ such that $g w \epsilon A$. 
Let $V=\underset{g \in G}{\cup} g \hat{A}$. Consider the sequence $\left\{K_{n}-V\right\}$. It satisfies (i), (ii), and using the fact that for $g \epsilon G, g V=V$, one sees that $\left\{K_{n}-V\right\}$ satisfies (iii). Since $K_{n}-V \varsubsetneqq K_{n}$ and $\left\{K_{n}\right\}$ is minimal in $\pi,\left\{K_{n}-V\right\} \notin \pi$, therefore, $\left\{K_{n}-V\right\}$ does not satisfy (iv), i.e., there exists $n_{0}$ such that $K_{n_{0}}-V=\phi$ which implies $K_{n_{0}} \subseteq V=\bigcup_{g \in G} g \hat{A}$. Since $K_{n_{0}}$ is compact, there exists $a_{1}, a_{2}, \ldots, a_{m} \epsilon G$ such that

$$
K_{n_{0}} \subset \bigcup_{i=1}^{m} a_{i} \hat{A}
$$

For each $n \geq n_{0}, g_{n} w \epsilon g_{n} K_{n+1} \subset K_{n} \subset K_{n_{0}}$, thus by $(*) g_{n} w \epsilon a_{i} \bar{A}$ for some $1 \leq i \leq m$. Then clearly, there exists $i_{0}, i_{0} \leq m$ such that $g_{n} w \epsilon a_{i_{0}} \bar{A}$ for infinitely many $n$. Thus the set $\{g \epsilon G: g w \epsilon \hat{A}\}$ is infinite. Therefore $w \epsilon R^{G}$.

REMARK: When $G=Z$, the above theorem is contained in Chou [1]. The idea of the proof of the above theorem follows from [1, Proposition 3.1].

LEMMA 2.3 (Chou [1]): The additive group of integers $Z$ contains an $R_{0}$-set.

For prime number $p$, let

$$
Z\left(p^{\infty}\right)=\left\{m / p^{n}: 0 \leq m<p^{n} ; m \epsilon Z, n=0, n=1,2, \ldots\right\}
$$

be the subgroup of $Q / Z$ generated by

$$
\left\{\frac{1}{p^{n}}: n=1,2, \ldots\right\} .
$$

LEMMA 2.4: For each prime number $p, A\left(p^{\infty}\right)$ contains an $R_{0}$-set.

PROOF: Let $G=A\left(p^{\infty}\right)$. Then $G$ is a subgroup of $Q / Z$. Note that $G$ can be written as $G=\bigcup_{n=1}^{\infty} H_{n}$ where $H_{1} \subset H_{2} \subset \ldots$ and each

$$
H_{n}=\left\{0, \frac{1}{p^{n}}, \frac{2}{p^{n}}, \ldots, p^{n}-\frac{1}{p^{n}}\right\} .
$$

is a cyclic group of order $p^{n}$. For convenience, we will write $H_{n}=\left\langle\frac{1}{p^{n}}\right\rangle$ (with the usual addition in $Q(\bmod Z))$. Then following Chou's construction for $Z$ [1], we define a sequence of subsets $E_{n}$ in $G$ by induction as follows:

$$
E_{1}=\left\{\frac{1}{p}\right\}, E_{n+1}=E_{n} \cup\left(E_{n}+\frac{1}{p^{(n+1)^{2}}}\right)
$$

Then

$$
\left|E_{n}\right|=2^{n-1} .
$$


Let

$$
\begin{aligned}
E=\bigcup_{n=1}^{\infty} E_{n} & =\left\{\frac{1}{p} ; \frac{1}{p}+\frac{1}{p 4} ; \frac{1}{p}+\frac{1}{p 9}, \frac{1}{p}+\frac{1}{p 4}+\frac{1}{p 9} ; \frac{1}{p}\right. \\
& +\frac{1}{p 16}, \frac{1}{p}+\frac{1}{p 4} \\
& +\frac{1}{p 16}, \frac{1}{p}+\frac{1}{p 9}+\frac{1}{p 16}, \frac{1}{p}+\frac{1}{p 4}+\frac{1}{p 9}+\frac{1}{p 16}, \frac{1}{p} \\
& +\frac{1}{p 25}, \frac{1}{p} \\
& \left.+\frac{1}{p 4}+\frac{1}{p 25}, \frac{1}{p}+\frac{1}{p 9}+\frac{1}{p 25}, \ldots\right\} .
\end{aligned}
$$

Consider the $F$-sequence $\left\{F_{n}\right\}$ in $G$ where $F_{n}=H_{n^{2}}$.

Then

$$
\left|F_{n}\right|=p^{n^{2}}
$$

and

$$
\bar{d}(E)=\ell \lim _{n} \frac{\left|E_{n}\right|}{\left|F_{n}\right|}=\ell \lim _{n} \frac{n-1}{p^{n^{2}}}=0 .
$$

Thus $E$ satisfies $\left(R_{0} \cdot 1\right)$.

We may choose a sequence of infinite subsets $C_{n}$ in $G$ such that

$$
\begin{aligned}
C_{1} & =\left\{\frac{1}{p}, \frac{1}{p}+\frac{1}{p 9}, \frac{1}{p}+\frac{1}{p 16}, \frac{1}{p}+\frac{1}{p 9}+\frac{1}{p 16}, \frac{1}{p}+\frac{1}{p 25},\right\} \\
& \left.=\frac{1}{p}+\frac{1}{p 9}+\frac{1}{p 25}, \frac{1}{p}+\frac{1}{p 16}+\frac{1}{p 25}, \frac{1}{p}+\frac{1}{p 9}+\frac{1}{p 16}+\frac{1}{p 25}, \frac{1}{p}+\frac{1}{p 36}, \ldots\right\} . \\
C_{2} & =\left\{\frac{1}{p}, \frac{1}{p}+\frac{1}{p 16}, \frac{1}{p}+\frac{1}{p 25}, \frac{1}{p}+\frac{1}{p 16}+\frac{1}{p 25}, \frac{1}{p}+\frac{1}{p 36}, \ldots\right\} \\
C_{3} & =\left\{\frac{1}{p}, \frac{1}{p}+\frac{1}{p 25}, \frac{1}{p}+\frac{1}{p 36}, \ldots\right\}, \text { and so on. }
\end{aligned}
$$

So

$$
E \supset C_{1} \supset C_{2} \supset \ldots
$$

and

$$
C_{n+1}+p^{-(n+1)^{2}} \subset C_{n} \text { for all } n
$$

Then by theorem $2.2, \bar{E}$ contains a recurrent point, and hence $E$ satisfies $\left(R_{0} \cdot 2\right)$.

REMARK: If $X$ is a discrete set and $Y$ is a subset of $X$, then we will consider $\beta Y$ as a subset of $\beta X$. Indeed $\beta Y$ is the closure of $Y$ in $\beta X$.

LEMMA 2.5: If an amenable group $G$ contains an infinite subgroup $H$ of infinite index, then $H$ is an $R_{0}$-set in $G$. 
PROOF: Since $[G: H]=\infty$, the number of disjoint cosets $\{t H\}$ is infinite. For any $\varphi \in \operatorname{LIM}(G)$, since $\varphi\left(\chi_{H}\right)=\varphi\left(\chi_{t H}\right)$ if $t_{1} H, t_{2} H, \ldots, t_{n} H$ are distinct cosets, then $\varphi\left(\chi_{t_{1} H \cup t_{2} H . \cup t_{n} H}\right)=\varphi\left(\chi_{t_{1} H}\right)+\ldots+\varphi\left(\chi_{t_{n} H}\right)=n \varphi\left(\chi_{t_{n} H}\right)<1$. Since $n$ is arbitrary, $\varphi\left(\chi_{H}\right)=0$ so $\bar{d}(H)=0$. Thus $H$ satisfies $\left(R_{0} \cdot 1\right)$. To see that $H$ satisfies $\left(R_{0} \cdot 2\right)$ in $G$, note that if $w \epsilon R^{H}$ and if $\cup$ is a neighbourhood of $w$ in $\beta G$, then $\cup \cap \bar{H}$ is also a neighbourhood of $w$ and $X^{\prime}=\{h \epsilon H: h w \epsilon \cup \cap \bar{H}\}$ is infinite. Therefore $\{g \epsilon G: g w \epsilon \cup\}$ ( $X)$ is infinite. Thus $w \epsilon R^{G}$. Since $\phi \neq A^{H} \subset R^{H}, R^{G} \cap \bar{H} \neq \phi$. Thus $H$ satisfies $\left(R_{0} \cdot 2\right)$ in $G$.

COROLLARY 2.6: If an amenable group $G$ is a direct product of infinitely many non-trivial subgroups $\left\{G_{\alpha}: \alpha \epsilon I\right\}$, then $G$ contains an $R_{0}$-set.

PROOF: To prove that $G$ contains an $R_{0}$-set, we constuct an infinite subgroup $H$ of $G$ of infinite index with the above lemma. Indeed, write $I=I_{1} \cup I_{2}$ such that both $I_{1}$ and $I_{2}$ are infinite, then $H=\pi\left\{G_{\alpha}: \alpha \epsilon I_{1}\right\}$ is what we want.

LEMMA 2.7: If an infinite suchgroup $H$ of an amenable group $G$ contains an $R_{0}$-set $A$, then $A$ is also an $R_{0}$-set in $G$.

PROOF: This is quite obvious since whenever $\bar{d}_{H}(A)=0$ then $\bar{d}_{G}(A)=0$ and $R^{H} \subset R^{G}$.

LEMMA 2.8: If $H$ is an amenable subgroup of an amenable group $G$ such that $G / H$ has an $R_{0}$-set $A^{\prime}$, then $\theta^{-1}\left(A^{\prime}\right)$ is an $R_{0}$-set in $G(\theta$ is the natural homomorphism of $G$ onto $G / H)$.

PROOF: Let $A=\theta^{-1}\left(A^{\prime}\right)$ and $G^{\prime}=G / H$. Then for each $\varphi \epsilon \operatorname{LIM}(G), \varphi\left(\chi_{A}\right)=$ $\varphi\left(\chi_{A} \cdot \theta\right)=\theta^{*} \varphi\left(\chi_{A^{\prime}}\right)=0$ since $\theta^{*} \varphi \epsilon \operatorname{LIM}\left(G^{\prime}\right)$ and $\bar{d}_{G^{\prime}}\left(A^{\prime}\right)=0$. Then $A$ satisfies $\left(R_{0} \cdot 1\right)$.

The natural homomorphism $\theta$ of $G$ onto $G^{\prime}$ can be extended to a continuous mapping of $\beta G$ onto $\beta G^{\prime}$. We will denote the extended mapping again by $\theta$. It is not hard to check that $\theta^{-1}\left(R^{G^{\prime}}\right) \subset R^{G}$, sine $A^{\prime} \cap \cap R^{G^{\prime}} \neq \phi$, and $A^{-} \cap R^{G} \neq \phi$ either. Thus $A$ satisfies $\left(R_{0} \cdot 2\right)$, and $A$ is an $R_{0}$-set as wanted.

Before providing our main result, we will need some definitions and some structure theorems for abelian groups. For the proofs see [6].

DEFINITION: Let $x \epsilon G$ and $n$ be an integer; $x$ is divisible by $n$ in case there is an element $y \epsilon G$ with $n y=x$. A group $G$ is divisible in case each $x \epsilon G$ is divisible by every $n>0$. 
A subgroup $S$ of $G$ is pure in $G$ in case $n G \cap S=n S$ for every integer $n$.

If $G$ is a periodic group, then a subgroup $B$ of $G$ is a basic subgroup of $G$ in the following cases: (i) $B$ is the direct sum of cyclic groups; (ii) $B$ is pure in $G$; (iii) $G / B$ is divisible.

\section{THEOREM 2.9:}

1. Every periodic group $G$ contains a basic subgroup $B$.

2. Every periodic group $G$ is a direct sum of $p$-groups.

3. Every periodic group is an extension of a direct sum of cyclic groups by a divisible group.

4. Every divisible subgroup $D$ is a direct sum of copies of $Q$ and of copies of $Z\left(p^{\infty}\right)$.

We are now ready to state and prove the main results of this paper.

THEOREM 2.10: If $G$ is an infinite abelian group, then $G$ contains an $R_{0}$-set.

PROOF: We will consider two cases:

1. $G$ : non-periodic. Then $G$ contains an infinite cyclic subgroup which can be regarded as the additive group of integers $Z$. Thus by lemmas 2.3 and 2.7 , there exists an $R_{0}$-set.

2. $G$ : periodic. Then by $2.9, G$ contains a basic subgroup $B$, so that $B$ is a direct sum of cyclic groups. Here we have two subcases:

(a) If $B$ is an infinite subgroup, by corollary 2.6 and lemma 2.7 , there exists an $R_{0}$-set.

(b) If $B$ is a finite subgroup, the question group $G / B$ is an infinite divisible that can be written as a direct sum of $\leq$ copies of $Z\left(P^{\infty}\right)$ (see 2.9). Consider one of these copies and apply lemmas 2.4 and 2.7 to get an $R_{0}$-set in $G$. Then the proof of the theorem is completed.

THEOREM 2.11: If $G$ is an infinite elementary amenable group, then $G$ contains an $R_{0}$-set.

PROOF: If $G$ is an abelian group, the theorem follows from the above one. If $G$ is not abelian, then by lemma 1.2, $G$ contains an infinite abelian subgroup $H$, and hence there exists an $R_{0}$-set $A$ in $H$. By lemma 2.7, $A$ is also an $R_{0}$-set in $G$. 
THEOREM 2.12: If $G$ is an infinite elementary amenable group, then

$$
R^{G} \supsetneqq A^{\bar{G}} \text { and } R^{G}-K^{-G} \neq \phi .
$$

The proof follows from the above theorem and proposition 2.1 .

COROLLARY 2.13: If $G$ is an infinite elementary amenable group, then

$$
R^{G} \varsubsetneqq W R^{G}
$$

This follows immediately from the above theorem and lemma 1.1.

CONJECTURE: Every infinite amenable group $G$ contains an $\boldsymbol{R}_{\mathbf{0}}$-set, and therefore

$$
A^{G} \varsubsetneqq R^{G} \quad \text { and } \quad R^{G}-K^{G} \neq \phi
$$

ACKNOWLEDGEMENT: The author would like to thank the referee for his comments.

\section{REFERENCES}

1. CHOU, C., Minimal sets, Recurrent points and Discrete orbits in $\beta N-N$, Illinois J. of Math., 22 \#1 (1978) pp. 54-63.

2. CHOU, C., Elementary amenable groups, Illinois J. of Math., 24 \# 3 (1980) pp. 396-407.

3. DAY, M. M. , Amenable semigroups; Illinois J. of Math., 1 (1957) pp. 509-544.

4. NASSAR, M., Recurrent and weakly recurrent point in $\beta G$. International J. of Math and Math Sciences, 9 No. 2(1986) pp. 319-322.

5. ROBINSON, J., Finiteness conditions and generalized soluble groups Part 1, Springer-Verlag, New York (1972).

6. ROTMAN, J., The Theory of Groups, second edition, Allyn and Bacon, Inc., Boston (1973). 


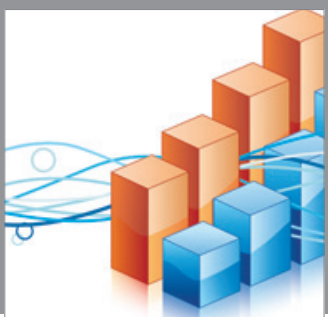

Advances in

Operations Research

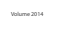

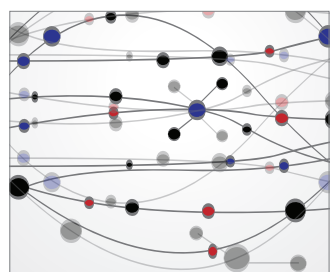

\section{The Scientific} World Journal
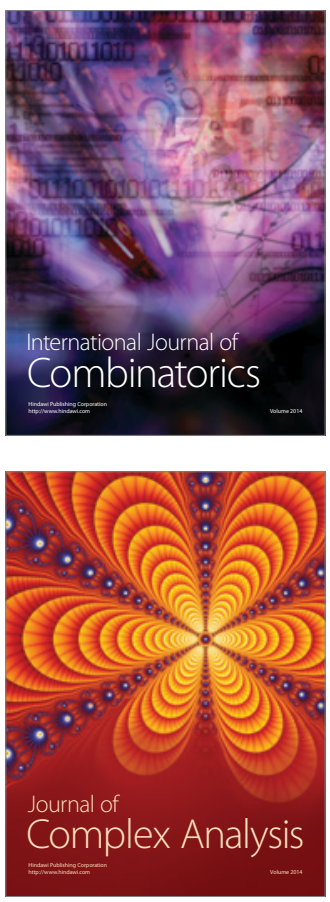

International Journal of

Mathematics and

Mathematical

Sciences
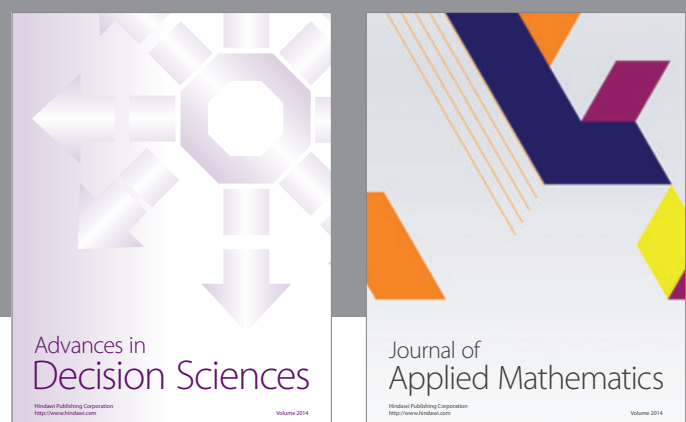

Journal of

Applied Mathematics
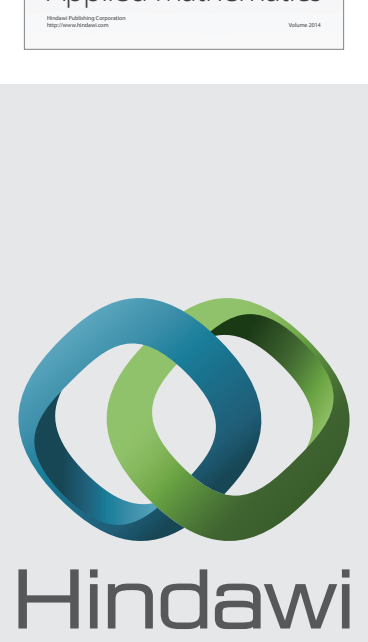

Submit your manuscripts at http://www.hindawi.com
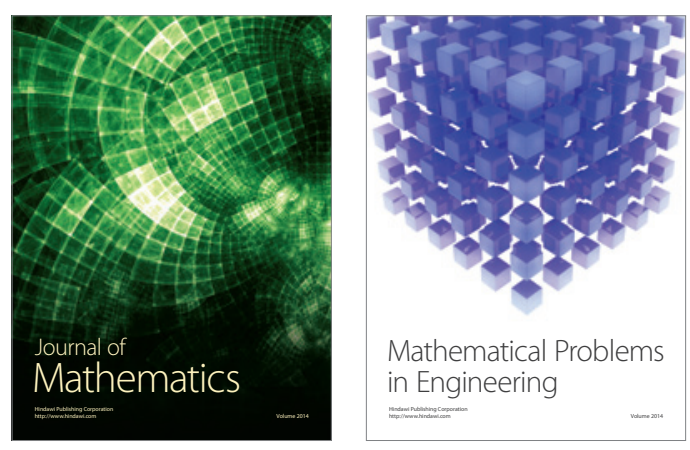

Mathematical Problems in Engineering
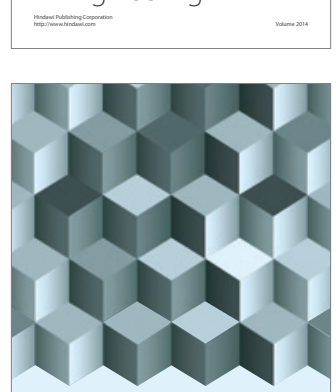

Journal of

Function Spaces
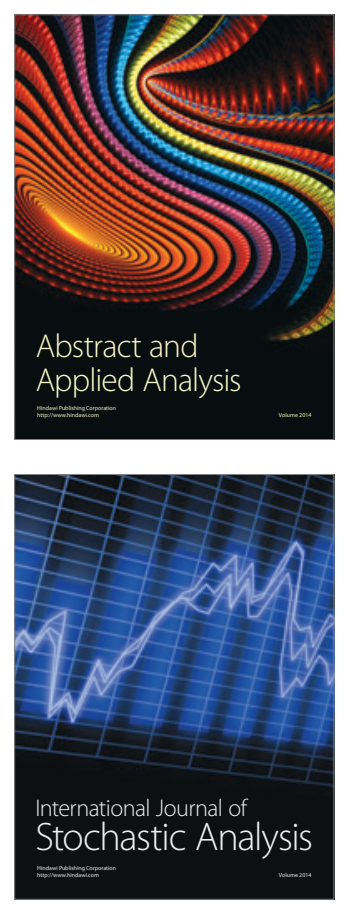

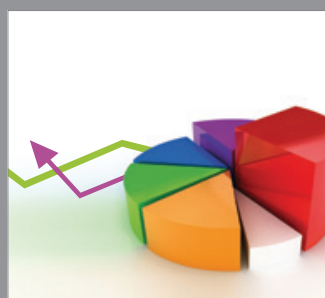

ournal of

Probability and Statistics

Promensencen
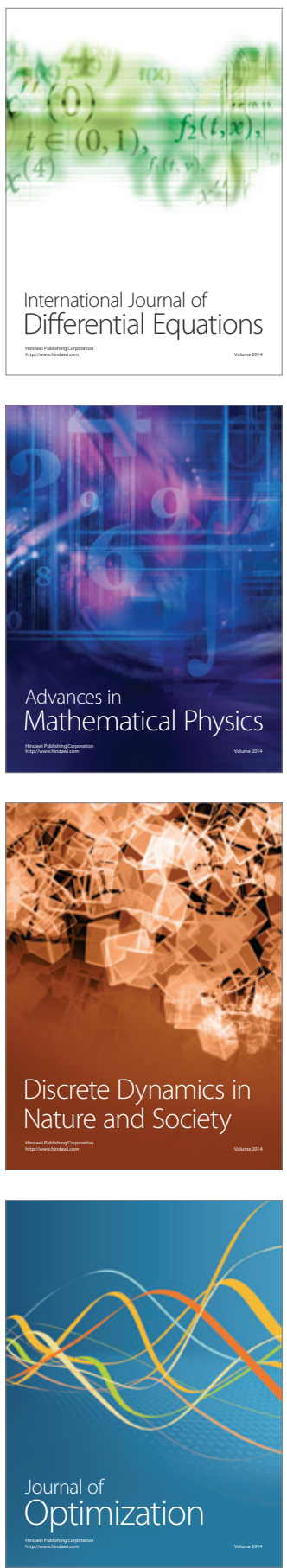